https://doi.org/10.15407/ujpe64.6.509

M.D. TOMCHENKO

Bogolyubov Institute for Theoretical Physics, Nat. Acad. of Sci. of Ukraine

(14b, Metrolohichna Str., Kyiv 03143,Ukraine; e-mail: mtomchenko@bitp.kiev.ua)

\title{
ELECTRIC FIELD AND ELECTRIC FORCES IN A SPONTANEOUSLY POLARIZED NONPOLAR ISOTROPIC DIELECTRIC
}

\begin{abstract}
Based on the microscopic Maxwell equations, we develop a method of description of the electric field in a spontaneously polarized isotropic nonpolar dielectric. We find the solution for the electric field $\boldsymbol{E}(\boldsymbol{r})$ for several typical examples. Moreover, we generalize Helmholtz's formula for the electric force acting on a volume element of a dielectric with regard for the contribution of the spontaneous polarization.
\end{abstract}

Keywords: spontaneous polarization, dielectric.

\section{Introduction}

As known, the polarization of dielectrics can be of two types: the polarization induced by an external electric field and the spontaneous one [1-7]. The spontaneous polarization is characteristic of ferroelectrics, pyroelectrics, and some of piezoelectrics. It can also arise in "customary" dielectrics. In particular, electric signals were observed in He II under torsion oscillations [8] and in standing half-waves of the second [911 ] and first [12] sounds. In those experiments, the electric field can be associated only with the spontaneous polarization, since ${ }^{4} \mathrm{He}$ atom possesses no intrinsic charge, dipole moment, or multipole moment.

Depending on the type of a dielectric, its atoms can be charged or neutral (the latter can be polar or nonpolar). For the ionic crystals, which can be piezoelectics, pyroelectrics, or ferroelectrics under certain conditions, the theory of spontaneous polarization is well developed [4, 6, 7, 13-15]. However, no such theory is available for nonpolar dielectrics (to our knowledge), though the properties of nonpolar dielectrics are more simple. In what follows, we try to remove this gap. We will consider only isotropic dielectrics.

(C) M.D. TOMCHENKO, 2019

ISSN 2071-0194. Ukr. J. Phys. 2019. Vol. 64, No. 6
For the nonpolar dielectrics, one needs to represent the total polarization $\mathbf{P}(\mathbf{r})$ as $\mathbf{P}_{i}(\mathbf{r})+\mathbf{P}_{s}(\mathbf{r})$ and to consider $\mathbf{P}_{s}$ and $\mathbf{P}_{i}$ self-consistently (here, $\mathbf{P}_{s}$ is the "bare" spontaneous polarization, and $\mathbf{P}_{i}$ is the induced polarization). This is seen from the following. In a nonpolar isotropic dielectric, the spontaneous polarization can arise due to sound, gravity, acceleration, and elastic deformation, since a directed concentration gradient appears in the medium in these cases. A nonpolar atom has no intrinsic dipole moment. However, two nonpolar atoms polarize each other. As a result, each atom acquires the dipole moment [16-18]

$\mathbf{d}=-D_{7}|e| \frac{a_{B}^{8}}{r^{7}} \mathbf{i}_{\mathbf{r}}$,

where $r$ is the distance between atoms, $\mathbf{i}_{\mathbf{r}}=\mathbf{r} / r$ is the unit vector directed to another atom, $a_{B}=\frac{\hbar^{2}}{m e^{2}}$ is the Bohr radius, and $D_{7}$ is an atomic constant. In view of this, the gradient of the particle number density $n(\mathbf{r})$ causes the bulk polarization of the medium

$\mathbf{P}_{s}(\mathbf{r})=\xi \nabla n(\mathbf{r})$.

The rough estimate on the basis of formula (1) gives $\xi=(7 / 3) d_{0} \bar{r}_{0}\left(n(\mathbf{r}) / n_{0}\right)^{a}$, where $a=2, d_{0}=$ 
$=-D_{7}|e| \frac{a_{B}^{8}}{\bar{r}_{0}^{7}}$, and $\bar{r}_{0}=n_{0}^{-1 / 3}$ is the mean interatomic distance in the unperturbed system. The more accurate analysis with the averaging of $\mathbf{d}$ over different configurations of atoms leads to $\xi \approx 7.5 \times$ $\times(7 / 3) d_{0} \bar{r}_{0}\left(n(\mathbf{r}) / n_{0}\right)^{a}$, where $a=1$ [19] (the number 7.5 is true only for He II, the sign of $\xi$ was obtained in [19] improperly). The collection of dipoles creating the polarization $\mathbf{P}_{s}(\mathbf{r})$ induces the field $\mathbf{E}(\mathbf{r})$. This field additionally polarizes the atoms and causes the induced polarization $\mathbf{P}_{i}(\mathbf{r})$. The latter was not taken into account in $\mathbf{P}_{s}(\mathbf{r})$ and also affects $\mathbf{E}(\mathbf{r})$. Thus, the electron shell of each atom is deformed by two different forces: first, due to the quantum mutual polarization of atoms and the density gradient, and, second, due to the field $\mathbf{E}$ in the medium. It is clear that, at small deformations, those two deformations should be independent of each other. At large deformations, we need to solve a quantum-mechanical problem of two nonpolar atoms in the external field E. Such problem has not been solved yet, as far as we know. In what follows, we consider the deformations to be small. In this case, the polarizations $\mathbf{P}_{s}$ and $\mathbf{P}_{i}$ should be considered separately. We believe that this is true for all mechanisms of spontaneous polarization of nonpolar (isotropic or anisotropic) dielectrics.

Based on the microscopic Maxwell equations, we will develop a method of determination of the polarization $\mathbf{P}(\mathbf{r})=\mathbf{P}_{s}(\mathbf{r})+\mathbf{P}_{i}(\mathbf{r})$ and the field $\mathbf{E}(\mathbf{r})$ for isotropic nonpolar dielectrics with the self-consistent account for $\mathbf{P}_{s}$ and $\mathbf{P}_{i}$. We will also show that the consideration of $\mathbf{P}_{s}$ changes the force acting on an element of the volume of a dielectric.

\section{Electric Field}

In the electrodynamics of continua (see [4], Chapters II, IV, IX), the electromagnetic field of isotropic dielectrics is described by the local (i.e., valid at each point of a medium) relations

$\mathbf{D}=\mathbf{E}+4 \pi \mathbf{P}=\varepsilon \mathbf{E}$,

$\mathbf{B}=\mathbf{H}+4 \pi \mathbf{M}=\mu \mathbf{H}$

and by the Maxwell equations in a medium:

$\operatorname{div} \mathbf{D}=4 \pi \rho_{f}$,

$\operatorname{rot} \mathbf{E}=-\frac{1}{c} \frac{\partial \mathbf{B}}{\partial t}$,

$\operatorname{div} \mathbf{B}=0$, $\operatorname{rot} \mathbf{H}=\frac{1}{c} \frac{\partial \mathbf{D}}{\partial t}+\frac{4 \pi \mathbf{j}_{f}}{c}$

Here, $\rho_{f}$ is the density of foreign charges, and $\mathbf{j}_{f}$ is the foreign current.

The spontaneous polarization of a dielectric arises due to the internal "nonelectric" mechanism causing the appearance of a set of dipoles in the medium; we will call them "spontaneous" dipoles. We will see below that, in order to describe a spontaneously polarized nonpolar isotropic dielectric, one needs to make some changes in Eqs. (3)-(8). Let us deduce the necessary equations from the microscopic Maxwell equations.

First, we find the formula for the polarization $\mathbf{P}(\mathbf{r})$, since the formulae available in the literature are not quite accurate sometimes. The density of polarization charges $\bar{\rho}(\mathbf{r})$, averaged over a physically infinitesimal volume, satisfies the relation

$\int_{V_{+}} \bar{\rho}(\mathbf{r}) d V=0$.

Therefore, $\bar{\rho}(\mathbf{r})$ can be presented in the form $\bar{\rho}(\mathbf{r})=$ $=-\operatorname{div} \mathbf{P}(\mathbf{r})$, where $\mathbf{P}=0$ outside the dielectric. Here, $V_{+}$is the volume confined inside the surface that covers the whole dielectric and oversteps its limits by an infinitesimal distance. In [4] (Chapt. 2, $\S 6), \mathbf{P}(\mathbf{r})$ is determined with the use of the following formulae:

$$
\begin{aligned}
& \int_{V_{+}} \mathbf{r} \bar{\rho}(\mathbf{r}) d V=-\int_{V_{+}} \mathbf{r}(\nabla \mathbf{P}(\mathbf{r})) d V= \\
& =-\oint_{S} \mathbf{r}(\mathbf{P}(\mathbf{r}) d \mathbf{S})+\int_{V_{+}} \mathbf{P}(\mathbf{r}) d V=\int_{V_{+}} \mathbf{P}(\mathbf{r}) d V .
\end{aligned}
$$

However, these formulae do not allow us to find $\mathbf{P}(\mathbf{r})$, since relation (10) does not yield $\mathbf{P}(\mathbf{r})=\mathbf{r} \bar{\rho}(\mathbf{r})$. Indeed, let the polarization be uniform: $\mathbf{P}(\mathbf{r})=\mathbf{P}$. Then $\bar{\rho}(\mathbf{r})=-\operatorname{div} \mathbf{P}=0$ inside the body. On the surface, $\mathbf{P}(\mathbf{r})$ decreases by jump to zero, and $\bar{\rho}(\mathbf{r})$ is singular. For such dielectric, the equality $\mathbf{P}(\mathbf{r})=\mathbf{r} \bar{\rho}(\mathbf{r})$ is violated at all points of the body. In this case, the integral $\int_{V_{+}} \mathbf{r} \bar{\rho}(\mathbf{r}) d V$ is defined by the surface part of $\bar{\rho}(\mathbf{r})$. These properties are natural, since a uniformly polarized body can be considered as two bodies that possess uniformly distributed $(+)$ and $(-)$ charges and are shifted relative to each other by an 
infinitesimal distance. The jump of $\bar{\rho}(\mathbf{r})$ on the surface is usually large for the nonuniform polarization as well. In this case, the equality $\mathbf{P}(\mathbf{r})=\mathbf{r} \bar{\rho}(\mathbf{r})$ should also be significantly violated. We note that the definition $\mathbf{P}(\mathbf{r})=\mathbf{r} \bar{\rho}(\mathbf{r})$ [4] was criticized previously in work [13].

In order to obtain the formula for $\mathbf{P}(\mathbf{r})$, we note that, for a polarized dielectric without foreign charges, $\rho(\mathbf{r})$ reads

$\rho(\mathbf{r})=\sum_{j=1}^{N}\left[q^{(j)} \delta\left(\mathbf{r}-\mathbf{r}_{j}\right)-q^{(j)} \delta\left(\mathbf{r}-\mathbf{r}_{j}-\mathbf{r}_{0}^{(j)}\right)\right]$,

where $N$ is the number of atoms in the dielectric. We set $q^{(j)}<0$ for all $j$. Then

$$
\int_{V_{+}} \mathbf{r} \rho(\mathbf{r}) d V=-\sum_{j=1}^{N} \mathbf{r}_{0}^{(j)} q^{(j)}=\sum_{j=1}^{N} \mathbf{d}_{j}=\int_{V_{+}} n(\mathbf{r}) \mathbf{d}(\mathbf{r}) d V
$$

where $n(\mathbf{r})$ is the microscopic density of dipoles, and $\mathbf{d}(\mathbf{r})$ is the dipole moment of a volume, which contains one atom and has the coordinate $\mathbf{r}$. Since $\int_{V_{+}} \rho(\mathbf{r}) d V=0$, we may write $\rho(\mathbf{r})=$ $=-\operatorname{div} \mathbf{P}_{\text {mic }}(\mathbf{r})$, where $\mathbf{P}_{\text {mic }}$ is a microscopic quantity. Similarly to (10), we obtain

$\int_{V_{+}} \mathbf{r} \rho(\mathbf{r}) d V=\int_{V_{+}} \mathbf{P}_{\text {mic }}(\mathbf{r}) d V$

The relations $\bar{\rho}(\mathbf{r})=-\operatorname{div} \mathbf{P}(\mathbf{r}) \quad$ and $\quad \bar{\rho}(\mathbf{r})=$ $=-\overline{\operatorname{div} \mathbf{P}_{\text {mic }}(\mathbf{r})}=-\operatorname{div} \overline{\mathbf{P}}_{\text {mic }}(\mathbf{r})$ yield $\mathbf{P}(\mathbf{r})=\overline{\mathbf{P}}_{\text {mic }}(\mathbf{r})$. Since equalities (12) and (13) hold for a dielectric of any shape, they yield finally

$\mathbf{P}_{\text {mic }}(\mathbf{r})=n(\mathbf{r}) \mathbf{d}(\mathbf{r}), \quad \mathbf{P}(\mathbf{r})=\overline{n(\mathbf{r}) \mathbf{d}(\mathbf{r})}$.

The possible jump of $\rho(\mathbf{r})$ on the surface does not violate equalities (14), since this jump on the righthand side of (12) is smeared over the whole volume. Therefore, the function $n(\mathbf{r}) \mathbf{d}(\mathbf{r})$ is smooth. The formula $\bar{\rho}(\mathbf{r})=-\operatorname{div} \mathbf{P}(\mathbf{r})$ sets $\mathbf{P}(\mathbf{r})$ to within $\operatorname{rot} \mathbf{g}(\mathbf{r})$, where $\mathbf{g}(\mathbf{r})$ is any function. Therefore, we can define $\mathbf{P}(\mathbf{r})$ in the infinite number of ways, but the chosen way must be consistent with the equation $\bar{\rho}(\mathbf{r})=-\operatorname{div} \mathbf{P}(\mathbf{r})$. For formulae (14), this holds. According to (14), $\mathbf{P}(\mathbf{r})$ is the dipole moment of a unit volume of the dielectric.

ISSN 2071-0194. Ukr. J. Phys. 2019. Vol. 64, No. 6
Relation (13) yields also $\mathbf{P}(\mathbf{r})=\overline{\mathbf{r} \rho(\mathbf{r})}$, though $\mathbf{P}_{\text {mic }}(\mathbf{r}) \neq \mathbf{r} \rho(\mathbf{r})$ (since $\rho(\mathbf{r})$ is a sum of $\delta$-functions, but $\mathbf{P}_{\text {mic }}(\mathbf{r})=n(\mathbf{r}) \mathbf{d}(\mathbf{r})$ is smoothly varied in space).

We now pass to the description of the spontaneous polarization. Consider an isotropic dielectric characterized in an external electric field by the dielectric permittivity $\varepsilon$. Let it contain a macroscopic number of spontaneous dipoles $\mathbf{d}_{s}^{(j)}$, and let it be surrounded by a dielectric with dielectric permittivity $\varepsilon_{2}$ (without the intrinsic electromagnetic field). We will write the equations only for the first dielectric and take the second one into account in boundary conditions. The spontaneous dipoles are associated with some average charge density $\bar{\rho}_{s}(\mathbf{r})$ and the polarization $\mathbf{P}_{s}$. These dipoles create the electric field, which polarizes the surrounding atoms (including the atoms, being the carriers of spontaneous dipoles). This leads to the appearance of induced charges with the average density $\bar{\rho}_{i}(\mathbf{r})$ and the induced polarization $\mathbf{P}_{i}(\mathbf{r})$. It is clear that, for the densities $\bar{\rho}_{s}(\mathbf{r})$ and $\bar{\rho}_{i}(\mathbf{r})$, the total charge is zero:

$\int_{V_{+}} \bar{\rho}_{s}(\mathbf{r}) d V=0, \quad \int_{V_{+}} \bar{\rho}_{i}(\mathbf{r}) d V=0$.

Similarly to the above analysis, (15) yield the formulae

$\bar{\rho}_{s}(\mathbf{r})=-\operatorname{div} \mathbf{P}_{s}(\mathbf{r}), \quad \bar{\rho}_{i}(\mathbf{r})=-\operatorname{div} \mathbf{P}_{i}(\mathbf{r})$,

$\mathbf{P}_{s}(\mathbf{r})=\overline{n_{s}(\mathbf{r}) \mathbf{d}_{s}(\mathbf{r})}, \quad \mathbf{P}_{i}(\mathbf{r})=\overline{n_{i}(\mathbf{r}) \mathbf{d}_{i}(\mathbf{r})}$,

where $n_{s}(\mathbf{r})$ and $n_{i}(\mathbf{r})$ are the microscopic densities of spontaneous and induced dipoles.

Next. The averaging of the microscopic Maxwell equation $\operatorname{div} \mathbf{E}_{\text {mic }}(\mathbf{r})=4 \pi \rho(\mathbf{r})$ over a physically infinitesimal volume gives the equation

$\operatorname{div} \mathbf{E}(\mathbf{r})=4 \pi \bar{\rho}(\mathbf{r})=4 \pi\left[\bar{\rho}_{s}(\mathbf{r})+\bar{\rho}_{i}(\mathbf{r})\right]$.

In view of $(16),(17)$, we can write (18) in the form

$\operatorname{div} \mathbf{D}(\mathbf{r})=0$,

where

$\mathbf{D}=\mathbf{E}+4 \pi\left(\mathbf{P}_{i}+\mathbf{P}_{s}\right)$.

As was mentioned above, the polarization $\mathbf{P}_{i}(\mathbf{r})$ is a local response of the medium to the field $\mathbf{E}(\mathbf{r})$. Therefore, the relation

$4 \pi \mathbf{P}_{i}(\mathbf{r})=(\varepsilon(\mathbf{r})-1) \mathbf{E}(\mathbf{r})$ 
should be satisfied analogously to the polarization of a dielectric by an external field $\mathbf{E}_{0}$. Here, $\varepsilon$ is the usual dielectric permittivity. Indeed, the response is independent of which force has created the field $\mathbf{E}_{0}$ at the given point of the dielectric: $\mathbf{E}_{0}$ can be created by free charges outside the system or by spontaneous charges inside the system. In both cases, the value of $\mathbf{P}_{i}$ must be the same. The resulting field is $\mathbf{E}=$ $=\mathbf{E}_{0}+\mathbf{E}_{i}$, where $\mathbf{E}_{i}$ is the field created by induced dipoles. Since $\mathbf{P}_{i}$ is identical in both cases, $\mathbf{E}$ should be also the same.

It is convenient to introduce the quantity

$\mathbf{D}_{i}=\mathbf{E}+4 \pi \mathbf{P}_{i}=\varepsilon \mathbf{E}$.

Then Eq. (18) takes the form

$\operatorname{div} \mathbf{D}_{i}(\mathbf{r})=4 \pi \bar{\rho}_{s}(\mathbf{r})$.

Thus, one should solve Eqs. (22), (23) with regard for the boundary conditions

$D_{n}=D_{2 n}, \quad \mathbf{E}_{t}=\mathbf{E}_{2 t}$

and the relation

$\mathbf{E}=-\nabla \varphi-\frac{1}{c} \frac{\partial \mathbf{A}}{\partial t}$

which follows from (6). In a stationary problem, the magnetic field is absent: $\mathbf{A}=0, \mathbf{B}=\operatorname{rot} \mathbf{A}=0$. If the spontaneous charges are moving, then a current $\mathbf{j}_{s}$ and a magnetic field arise. The charge moving with a velocity $\mathbf{v}$ creates the potential $\varphi$ and the vector potential $\mathbf{A}=\varphi \mathbf{v} / c[20]$ (in the immovable reference system). The last relation indicates that the magnetic field is weak for the processes, which are slow as compared with the electromagnetic wave. Therefore, for the stationary and slow processes, we may set $\partial \mathbf{A} / \partial t=0$ in (25), and Eq. (23) takes the form of the Poisson equation

$\triangle \varphi=-4 \pi \bar{\rho}_{s} / \varepsilon$.

We neglect the nonuniformity of $\varepsilon$, which is justified for uniform and weakly nonuniform fields.

Let the spontaneous dipoles $\mathbf{d}_{s}^{(j)}=\left|q_{0}^{(j)}\right| \mathbf{r}_{0}^{(j)}\left(q_{0}^{(j)}<\right.$ $<0)$ be distributed in the dielectric. The average density of effective spontaneous charges reads

$\bar{\rho}_{s}=\left\langle\sum_{j=1}^{N_{s}}\left[q_{0}^{(j)} \delta\left(\mathbf{r}-\mathbf{r}_{j}\right)-q_{0}^{(j)} \delta\left(\mathbf{r}-\mathbf{r}_{j}-\mathbf{r}_{0}^{(j)}\right)\right]\right\rangle$, where $N_{s}$ is the number of spontaneous dipoles, $\mathbf{r}_{j}$ and $\mathbf{r}_{j}+\mathbf{r}_{0}^{(j)}$ are the coordinates of the effective charges $q_{0}^{(j)},-q_{0}^{(j)}$ of the $j$-th dipole, and $\langle f\rangle \equiv \bar{f}$. The solution of Eqs. (26), (27) is known:

$\varphi(\mathbf{r})=\left\langle\sum_{j=1}^{N_{s}}\left[\frac{q_{0}^{(j)}}{\varepsilon\left|\mathbf{r}-\mathbf{r}_{j}\right|}-\frac{q_{0}^{(j)}}{\varepsilon\left|\mathbf{r}-\mathbf{r}_{j}-\mathbf{r}_{0}^{(j)}\right|}\right]\right\rangle$.

For the points $\mathbf{r}$ far from the spontaneous dipoles $\left(\left|\mathbf{r}-\mathbf{r}_{j}\right| \gg r_{0}^{(j)}\right)$, we can make expansion in $\mathbf{r}_{0}^{(j)}$. As a result, we have

$$
\begin{aligned}
& \varphi(\mathbf{r})=\left\langle\sum_{j=1}^{N_{s}} \frac{\mathbf{d}_{s}^{(j)}\left(\mathbf{r}-\mathbf{r}_{j}\right)}{\varepsilon\left|\mathbf{r}-\mathbf{r}_{j}\right|^{3}}\right\rangle= \\
& =\left\langle\int_{V} d \dot{\mathbf{r}} \frac{n_{s}(\dot{\mathbf{r}}) \mathbf{d}_{s}(\dot{\mathbf{r}})(\mathbf{r}-\dot{\mathbf{r}})}{\varepsilon|\mathbf{r}-\dot{\mathbf{r}}|^{3}}\right\rangle= \\
& =\int_{V} d \dot{\mathbf{r}} \frac{\overline{n_{s}(\dot{\mathbf{r}}) \mathbf{d}_{s}(\dot{\mathbf{r}})(\mathbf{r}-\dot{\mathbf{r}})}}{\varepsilon|\mathbf{r}-\dot{\mathbf{r}}|^{3}}=\int_{V} d \dot{\mathbf{r}} \frac{\mathbf{P}_{s}(\dot{\mathbf{r}})(\mathbf{r}-\dot{\mathbf{r}})}{\varepsilon|\mathbf{r}-\dot{\mathbf{r}}|^{3}},
\end{aligned}
$$

where $V$ is the volume of the dielectric.

Formula (29) without $\varepsilon$ in the denominator is well known [5]. The quantity $\varepsilon$ in (29) takes the polarization $\mathbf{P}_{i}$ into account. Thus, taking $\mathbf{P}_{i}$ into consideration leads to the replacements $\mathbf{d}_{s} \rightarrow \mathbf{d}_{s} / \varepsilon$, $\mathbf{P}_{s} \rightarrow \mathbf{P}_{s} / \varepsilon$ in (29). This change has a simple physical meaning: it shows that the medium weakens the field of a dipole by $\varepsilon$ times. In view of this reasoning, formula (29) was intuitively guessed in [19,21].

For the uniform spontaneous polarization, we have $\mathbf{P}_{s}=\left(N_{s} / V\right) \mathbf{d}_{s}=\bar{n}_{s} \mathbf{d}_{s}$ and

$\varphi(\mathbf{r})=\int_{V} d \dot{\mathbf{r}} \frac{\mathbf{P}_{s}(\mathbf{r}-\dot{\mathbf{r}})}{\varepsilon|\mathbf{r}-\dot{\mathbf{r}}|^{3}}=-\mathbf{P}_{s} \nabla_{\mathbf{r}} \int_{V} \frac{d \dot{\mathbf{r}}}{\varepsilon|\mathbf{r}-\dot{\mathbf{r}}|}$.

With regard for the formula [22]

$\nabla(\mathbf{F G})=(\mathbf{F} \nabla) \mathbf{G}+(\mathbf{G} \nabla) \mathbf{F}+\mathbf{F} \times(\nabla \times \mathbf{G})+$

$+\mathbf{G} \times(\nabla \times \mathbf{F})$,

we find

$\mathbf{E}(\mathbf{r})=-\nabla_{\mathbf{r}} \varphi(\mathbf{r})=\left(\mathbf{P}_{s} \nabla_{\mathbf{r}}\right) \nabla_{\mathbf{r}} f(\mathbf{r})$,

$f(\mathbf{r})=\int_{V} \frac{d \dot{\mathbf{r}}}{\varepsilon|\mathbf{r}-\dot{\mathbf{r}}|}$

ISSN 2071-0194. Ukr. J. Phys. 2019. Vol. 64, No. 6 
from (30). Formulae (29) and (32) give the solution for the field $\mathbf{E}(\mathbf{r})$ created by a spontaneously polarized isotropic dielectric of volume $V$ surrounded by vacuum. It is seen from (32) that $\mathbf{E}(\mathbf{r})=\operatorname{const} \mathbf{P}_{s}$ only in particular cases, for example, if $f(\mathbf{r})=b_{0}+$ $+\mathbf{b}_{1} \mathbf{r}+b_{2} \mathbf{r}^{2}$ or if $\mathbf{P}_{s}=P_{s} \mathbf{i}_{z}, f(\mathbf{r})=b_{0}+b_{1} z+b_{2} z^{2}$. The symmetry-based reasoning implies that, for a finite $V$, the first case with $\mathbf{b}_{1}=0$ is possible only for the dielectrics with shape of a ball (the case with $\mathbf{b}_{1} \neq 0$ corresponds probably to an ellipsoid), but the second case is impossible for a three-dimensional system. In other words, $\mathbf{E}(\mathbf{r})$ is not codirected with $\mathbf{P}_{s}$ in the general case. Moreover, $\mathbf{E}$ can be nonuniform, when $\mathbf{P}_{s}$ is uniform. We have verified these properties for a cylindrical dielectric with $\mathbf{P}_{s}=P_{s} \mathbf{i}_{z}$ (see also Sec. 6.1). These properties suggest that, for an isotropic spontaneously polarized dielectric, the relationship between the electric induction

$\mathbf{D}=\mathbf{D}_{i}+4 \pi \mathbf{P}_{s}=\varepsilon \mathbf{E}+4 \pi \mathbf{P}_{s}$

and the strength $\mathbf{E}$ takes generally the tensor form:

$D_{j}=\sum_{l} \tilde{\varepsilon}_{j l} E_{l}, \quad \tilde{\varepsilon}_{j l}=\delta_{j l} \varepsilon+\zeta_{j l}$.

According to Eqs. (29) and (32), the field $\mathbf{E}(\mathbf{r})$ is connected with $\mathbf{P}_{s}(\mathbf{r})$ by the relation $\mathbf{E}(\mathbf{r})=\hat{\varsigma}(\mathbf{r}) \mathbf{P}_{s}(\dot{\mathbf{r}})$, where $\hat{\varsigma}(\mathbf{r})$ is a linear integro-differential operator. In this case, we can write $E_{j}(\mathbf{r})=\sum_{l} \varsigma_{j l}(\mathbf{r}) P_{s, l}(\mathbf{r})$ and $\zeta_{j l}(\mathbf{r})=4 \pi \varsigma_{j l}^{-1}(\mathbf{r})$, where $\varsigma_{j l}(\mathbf{r})$ is the matrix of eigenvalues of the operator $\hat{\varsigma}(\mathbf{r})$. We note that the tensor $\zeta_{j l}$ characterizes the spontaneous polarization and is physically different from the tensor of dielectric permittivity $\varepsilon_{j l}$. We also note that $\tilde{\varepsilon}_{j l}$ is not the tensor of dielectric permittivity as well, though it contains $\varepsilon$. Indeed, the quantity $\varepsilon_{j l}$ characterizes the response $\mathbf{P}_{i}$ of the system to an external field $\mathbf{E}$. On the contrary, the quantity $\zeta_{j l}$ allows one to determine the field $\mathbf{E}(\mathbf{r})$ created by the polarization $\mathbf{P}_{s}(\mathbf{r})$. Moreover, $\varepsilon_{j l}$ is a local quantity. However, $\zeta_{j l}$ is a nonlocal quantity, since its value is determined by the distribution of the spontaneous polarization in the whole volume of the dielectric and by the boundary conditions. For an anisotropic dielectric polarized by an external electric field, the principal values of the tensor $\varepsilon_{j l}$ must be $\geq 1$ [4]. However, for $\zeta_{j l}$ and $\tilde{\varepsilon}_{j l}$, such restriction is absent. The tensor character of $\zeta_{j l}$ is a consequence of the fact that the field $\mathbf{E}(\mathbf{r})$ (32) created by spontaneous dipoles is not codirected with $\mathbf{P}_{s}(\mathbf{r})$ in the general case. If $\mathbf{E}(\mathbf{r})$ and $\mathbf{P}_{s}(\mathbf{r})$ are codirected, then $\zeta(\mathbf{r})$ is a scalar: $\zeta_{j l}(\mathbf{r})=\delta_{j l} \zeta(\mathbf{r}), 4 \pi \mathbf{P}_{s}=\zeta(\mathbf{r}) \mathbf{E}(\mathbf{r})$.

We remark that the nonlocal connection between the intrinsic field $\mathbf{E}(\mathbf{r})$ and $\mathbf{P}(\mathbf{r})$ was derived previously for ferroelectrics [15, 23-25]. In this case, the solutions $[15,23,25]$ have the local form $\mathbf{E}(\mathbf{r})=$ const $\mathbf{P}(\mathbf{r})$ (the nonlocality is hidden due to the one-dimensional geometry and/or the uniformity of $\mathbf{P}$ ). Ferroelectrics were considered $[15,23-25]$ without the separation of the total polarization $\mathbf{P}$ into $\mathbf{P}_{s}$ and $\mathbf{P}_{i}$.

In ferroelectrics, the distribution of the polarization is regulated by the minimum of the thermodynamic potential $\Phi[4,7,14,15,26]$. Therefore, it should consider namely the total polarization $\mathbf{P}(\mathbf{r})$ to be known. In such case, the above formulae are applicable, if we change $\bar{\rho}_{s} \rightarrow \bar{\rho}, \mathbf{P}_{s}(\mathbf{r}) \rightarrow \mathbf{P}(\mathbf{r})$, and $\bar{\rho}_{i}, \mathbf{P}_{i}(\mathbf{r}) \rightarrow 0$. This is equivalent to one change: $\varepsilon \rightarrow 1$. For the piezoelectrics, one can represent $\mathbf{P}(\mathbf{r})$ in the form of the deformational and induced parts [similarly to Eq. (40) below, but in the tensor form] $[4,6]$. In this case, the second term affects the first one by means of the equations of elastic equilibrium. For the nonpolar dielectrics, the influence of $\mathbf{P}_{i}$ on $\mathbf{P}_{s}$ is negligible (see the following section). Such difference is apparently due to the fact that the force acting on the volume element is $F \sim E$ for a piezoelectric and $F \sim E^{2}$ for a nonpolar dielectric (in this case, the field $E$ is weak; here, we talk about the electric part of $\mathbf{F})$.

Thus, in order to find the field $\mathbf{E}(\mathbf{r})$ in a spontaneously polarized nonpolar isotropic dielectric, we need to solve Eqs. (22), (23) with the boundary conditions (24), in which $\mathbf{D}$ is given by formula (33). If the dielectric is surrounded by vacuum, then Eq. (29) gives the exact solution. In this case, the boundary conditions (24) are satisfied automatically.

Let us turn to the Maxwell equations (5)-(8). We noted above that in the presence of the spontaneous polarization one should solve Eq. (23) (with the replacement $\bar{\rho}_{s}(\mathbf{r}) \rightarrow \bar{\rho}_{s}(\mathbf{r})+\rho_{f}(\mathbf{r})$, if foreign charges are present) instead of Eq. (5). In this case, Eq. (23) is simply another form of Eq. (5). Equations (6) and (7) remain the same. Equation (8) can be verified similarly to the analysis in [4] (Chapt. IX, §75). We conclude that Eq. (8) is valid. In this case, $\mathbf{D}$ is given by formula (33). However, while solving Eq. (8), we should separate $\mathbf{P}_{s}$ from $\mathbf{D}$ by means of the replacements $\mathbf{D} \rightarrow \mathbf{D}_{i}$ and $\mathbf{j}_{f} \rightarrow \mathbf{j}_{f}+\overline{\mathbf{j}}_{s}$, where $\overline{\mathbf{j}}_{s}=\overline{\rho_{s} \mathbf{v}_{s}}$ is the current related to spontaneous dipoles. Thus, the Maxwell equations (5)-(8) conserve formally their 
validity. However, while solving them, it is necessary to separate $\mathbf{P}_{s}$ from $\mathbf{D}$, since the spontaneous dipoles are the source of the field. In addition, $\mathbf{D}$ is given by formulae (33), (34) instead of (3).

In experiments, the potential $\varphi(\mathbf{r})$ is measured during a macroscopic time. For such time interval, a configuration of spontaneous dipoles change a huge number of times. Therefore, we need to average $\varphi(\mathbf{r})$ over the time. According to Gibbs [27], the average over the time can be replaced by the average over the ensemble. Quantum statistics gives the following formula for the average over the ensemble [28]:

$$
\langle\hat{\varphi}\rangle_{\mathrm{ens}}=\int d \Omega Z^{-1} \sum_{n} e^{-E_{n} / k_{\mathrm{B}} T} \Psi_{n}^{*} \hat{\varphi} \Psi_{n}
$$

where $Z=\sum_{n} e^{-E_{n} / k_{\mathrm{B}} T}, \hat{\varphi}$ is given by $(29), E_{n}$ is the energy of the system in the $n$-th state, and $\left\{\Psi_{n}\right\}$ is the complete collection of wave functions of the system. The operator $\hat{\varphi}$ and the functions $\Psi_{n}$ should be written in terms of the coordinates of the nucleus and electrons of each atom. Thus, the polarization $\mathbf{P}_{s}(\dot{\mathbf{r}})$ in (29) should be additionally averaged statistically. Then $\mathbf{P}_{s}(\dot{\mathbf{r}})=\left\langle\overline{n_{s}(\dot{\mathbf{r}}) \mathbf{d}_{s}(\dot{\mathbf{r}})}\right\rangle_{\mathrm{ens}}=$ $=\overline{\left\langle n_{s}(\dot{\mathbf{r}}) \mathbf{d}_{s}(\dot{\mathbf{r}})\right\rangle_{\mathrm{ens}}}$.

\section{Mechanical Response of a Dielectric to an External Electric Field}

In Section 2, we assumed that the quantity $\mathbf{P}_{s}$ is fixed and can be obtained with regard for the mechanism of spontaneous polarization. The fixity of $\mathbf{P}_{s}$ means that the field $\mathbf{E}(\mathbf{r})$ created by dipoles of the medium has no effect on $\mathbf{P}_{s}(\mathbf{r})$. We now find the influence of the field $\mathbf{E}$ on the value of $\mathbf{P}_{s}$, which allows us to write the complete system of equations for $\mathbf{P}(\mathbf{r})$ and $\mathbf{E}(\mathbf{r})$. The polarization $\mathbf{P}_{s}(\mathbf{r})$ arises at $\nabla n \neq 0$. Therefore, we should determine the influence of the field $\mathbf{E}$ on the density $n(\mathbf{r})$. The motion of the ideal fluid is described by the Euler equation

$\rho D \mathbf{v} / D t \equiv \rho \partial \mathbf{v} / \partial t+\rho(\mathbf{v} \nabla) \mathbf{v}=\mathbf{F}$

and by the equation of continuity

$\partial \rho / \partial t+\operatorname{div}(\rho \mathbf{v})=0$

where

$\mathbf{F}=-\nabla p+\mathbf{F}^{n m}$,
$\mathbf{F}^{n m}$ is a nonmechanical (usually external) force per unit volume, $\rho=m n$, and $m$ is the mass of an atom of the dielectric. In the presence of the electric field, $\mathbf{F}^{n m}$ is approximately the force, with which the field $\mathbf{E}$ acts on the dipole moment of a unit volume. Since the force acting on the charge $q$ is $q \mathbf{E}+[\mathbf{v} \times \mathbf{H}] q / c$, we find, by neglecting the magnetic field,

$\mathbf{F}^{n m} \approx(\mathbf{P} \nabla) \mathbf{E}, \quad \mathbf{E}(\mathbf{r})=\mathbf{E}^{\mathrm{own}}(\mathbf{r})+\mathbf{E}^{\mathrm{ext}}(\mathbf{r})$.

Here, $\mathbf{E}^{\text {own }}$ is the field induced by the dipoles of the dielectric, and $\mathbf{E}^{\text {ext }}$ is the external field. According to the above analysis, we get

$\mathbf{P}=\mathbf{P}_{s}+\mathbf{P}_{i}=\xi \nabla n(\mathbf{r})+\frac{\varepsilon-1}{4 \pi} \mathbf{E}$.

Therefore,

$\mathbf{F}^{n m} \approx \xi(\nabla n \nabla) \mathbf{E}+\frac{\varepsilon-1}{8 \pi} \nabla E^{2}$.

Without an external field $\left(\mathbf{E}^{\text {ext }}=0\right)$, we have $\left|F^{n m}\right| \ll|\nabla p|$. Indeed, under the spontaneous polarization, the relation $E \sim P \sim P_{s}$ usually holds (see Appendix). Setting $E=P_{s}$ in (41) and using formula (2) with $\xi=(7 / 3) d_{0} \bar{r}_{0}\left(n(\mathbf{r}) / n_{0}\right)^{2}$, we get $\left|F^{n m}\right| /|\nabla p| \sim 10^{-16} \bar{r}_{0}^{2}\left|(\triangle n) / n+2((\nabla n) / n)^{2}\right|$ for HeII (for other dielectrics, $\left|F^{n m}\right| /|\nabla p|$ can be different by several orders of magnitude). This is a negligible value, since $\bar{r}_{0}^{2}\left|(\triangle n) / n+2((\nabla n) / n)^{2}\right| \lesssim 1$ for real systems. From the physical point of view, the smallness of $\left|F^{n m}\right| /|\nabla p|$ is related to the small value of the mutual polarization of two nonpolar atoms (e.g., the electron shell of a He II atom is elongated due to the interaction with adjacent atoms by $\sim 10^{-5} \AA$ ) and to that $F^{n m} \sim E^{2}$. Therefore, we may neglect the term $\mathbf{F}^{n m}$ in Eqs. (36) and (38). Hence, for $\mathbf{E}^{\text {ext }}=0$, the influence of the field $\mathbf{E}$ on the velocity of an element of the fluid and on the density can be neglected. Therefore, we can consider the polarization $\mathbf{P}_{s}$ in Eq. (29) to be a fixed quantity depending on $\nabla n$ and independent of $\mathbf{E}$ (as was assumed in Section 2).

The more accurate derivation of the expression for a force is complicated and gives Helmholtz's formula (see [4], Chap. II):

$\mathbf{F}^{n m}=\frac{1}{8 \pi} \nabla\left[E^{2} \rho\left(\frac{\partial \varepsilon}{\partial \rho}\right)_{T}\right]-\frac{E^{2}}{8 \pi} \nabla \varepsilon$.

This formula is obtained for the polarization by the external field $(\mathbf{D}=\varepsilon \mathbf{E})$ and does not involve the spontaneous polarization $\mathbf{P}_{s}$.

ISSN 2071-0194. Ukr. J. Phys. 2019. Vol. 64, No. 6 
For a spontaneously polarized dielectric, we should consider also the polarization $\mathbf{P}_{s}$, since

$\mathbf{D}=\varepsilon \mathbf{E}+4 \pi \mathbf{P}_{s}$

(see Eq. (33)). Let us determine the force $\mathbf{F}$ with regard for the contribution of $\mathbf{P}_{s}$. We will apply the analysis made in [4] and will present only the changes caused by $\mathbf{P}_{s}$. As for the details, see [4], $\S 15,16$. We are based on the formulae obtained for a liquid dielectric [4]:

$F_{i}=\sum_{k} \frac{\partial \sigma_{i k}}{\partial x_{k}}$,

$\sigma_{i k}=\left[\tilde{F}-\left.\rho \frac{\partial \tilde{F}}{\partial \rho}\right|_{\mathbf{E}, T}\right] \delta_{i k}+\frac{E_{i} D_{k}}{4 \pi}$,

$d \tilde{F}=-S d T+\zeta d \rho-\frac{1}{4 \pi} \mathbf{D} d \mathbf{E}$.

Here, $\sigma_{i k}$ is the stress tensor, $\tilde{F}$ is the free energy per unit volume, and $\zeta$ is the chemical potential per unit mass.

Since $\mathbf{P}_{s}$ is independent of $\mathbf{E}$, relations (43) and (46) yield

$\tilde{F}=\tilde{F}_{0}(\rho, T)-\frac{\varepsilon E^{2}}{8 \pi}-\mathbf{P}_{s} \mathbf{E}$.

The account for $\mathbf{P}_{s}$ according to (43), (47) gives the following addition to $\sigma_{i k}$ :

$\sigma_{i k}^{s}=\left[-\mathbf{P}_{s} \mathbf{E}+\left(\left.\rho \frac{\partial \mathbf{P}_{s}}{\partial \rho}\right|_{T}\right) \mathbf{E}\right] \delta_{i k}+E_{i} P_{s, k}$.

However, the complete tensor $\sigma_{i k}$ for such solution has no symmetry $\sigma_{i k}=\sigma_{k i}$, which should hold [4, 29]. In order to restore this symmetry, we take into account that the spontaneous polarization $\mathbf{P}_{s}(\mathbf{r})$ separates a local axis in the medium and leads to the anisotropy (though the distribution of atoms is isotropic). The anisotropy is also indicated by formula (34). By means of a rotation of the coordinate system, the tensor $\tilde{\varepsilon}_{j l}$ can be diagonalized, which separates also definite axes. To account for such anisotropy, we can use the reasoning for the crystal axes (see [4], $\S 16)$. The result consists in the requirement to make change

$E_{i} D_{k} \rightarrow\left(E_{i} D_{k}+E_{k} D_{i}\right) / 2$

in (45). Then relation (45) becomes

$\sigma_{i k}=\left[\tilde{F}-\left.\rho \frac{\partial \tilde{F}}{\partial \rho}\right|_{\mathbf{E}, T}\right] \delta_{i k}+\frac{E_{i} D_{k}+E_{k} D_{i}}{8 \pi}$, and $\sigma_{i k}^{s}$ takes the form

$\sigma_{i k}^{s}=\left[-\mathbf{P}_{s} \mathbf{E}+\left(\left.\rho \frac{\partial \mathbf{P}_{s}}{\partial \rho}\right|_{T}\right) \mathbf{E}\right] \delta_{i k}+\frac{E_{i} P_{s, k}+E_{k} P_{s, i}}{2}$.

According to Introduction, $\mathbf{P}_{s}(\mathbf{r})=$ const $n^{a} \nabla n$. Therefore, we set $\rho \frac{\partial \mathbf{P}_{s}}{\partial \rho}=a \mathbf{P}_{s}$. Then

$\sigma_{i k}^{s}=(a-1) \mathbf{P}_{s} \mathbf{E} \delta_{i k}+\frac{E_{i} P_{s, k}+E_{k} P_{s, i}}{2}$.

By (44), this gives the additional force

$\mathbf{F}_{s}=(a-1) \nabla\left(\mathbf{P}_{s} \mathbf{E}\right)+\frac{1}{2} \mathbf{E} \operatorname{div} \mathbf{P}_{s}+$

$+\frac{1}{2}\left(\mathbf{P}_{s} \nabla\right) \mathbf{E}+\frac{1}{2} \mathbf{P}_{s} \operatorname{div} \mathbf{E}+\frac{1}{2}(\mathbf{E} \nabla) \mathbf{P}_{s}$.

By assuming $\mathbf{D}=\varepsilon \mathbf{E}$, the formula

$F_{j}=\frac{\partial}{\partial x_{j}}\left[-p+\left.\frac{E^{2}}{8 \pi} \rho \frac{\partial \varepsilon}{\partial \rho}\right|_{T}\right]-\frac{E^{2}}{8 \pi} \frac{\partial \varepsilon}{\partial x_{j}}+$

$+\frac{1}{4 \pi}\left[-\frac{\varepsilon}{2} \frac{\partial E^{2}}{\partial x_{j}}+\sum_{k} \frac{\partial}{\partial x_{k}}\left(\varepsilon E_{j} E_{k}\right)\right]$

was obtained in [4]. Further, the property $\operatorname{div} \mathbf{D}=$ $=\sum_{k} \frac{\partial}{\partial x_{k}}\left(\varepsilon E_{k}\right)=0$ was used in [4]. This property yields $-\frac{\varepsilon}{2} \frac{\partial E^{2}}{\partial x_{j}}+\sum_{k} \frac{\partial}{\partial x_{k}}\left(\varepsilon E_{j} E_{k}\right)=0$ in (54). In our case, relation (43) leads to the formula $\operatorname{div} \mathbf{D}=$ $=\sum_{k} \frac{\partial}{\partial x_{k}}\left(\varepsilon E_{k}\right)+4 \pi \sum_{k} \frac{\partial}{\partial x_{k}}\left(P_{s, k}\right)=0$. Therefore,

$-\frac{\varepsilon}{2} \frac{\partial E^{2}}{\partial x_{j}}+\sum_{k} \frac{\partial}{\partial x_{k}}\left(\varepsilon E_{j} E_{k}\right)=-4 \pi E_{j} \operatorname{div} \mathbf{P}_{s}$.

Formulae (53), (54), and (55) yield the solution:

$\mathbf{F}=\nabla\left[-p+\left.\frac{E^{2}}{8 \pi} \rho \frac{\partial \varepsilon}{\partial \rho}\right|_{T}\right]-\frac{E^{2}}{8 \pi} \nabla \varepsilon+$ $+(a-1) \nabla\left(\mathbf{P}_{s} \mathbf{E}\right)-\frac{1}{2} \mathbf{E} \operatorname{div} \mathbf{P}_{s}+\frac{1}{2} \mathbf{P}_{s} \operatorname{div} \mathbf{E}+$

$+\frac{1}{2}\left(\mathbf{P}_{s} \nabla\right) \mathbf{E}+\frac{1}{2}(\mathbf{E} \nabla) \mathbf{P}_{s}$.

Using relations (31), $\operatorname{rot} \mathbf{E}=0, \operatorname{rot} \mathbf{P}_{s}=0$ (since $\mathbf{P}_{s}(\mathbf{r})=$ const $\left.\nabla n^{a+1}\right)$, and $[22]$

$\operatorname{rot}(\mathbf{F} \times \mathbf{G})=(\mathbf{G} \nabla) \mathbf{F}-(\mathbf{F} \nabla) \mathbf{G}+\mathbf{F} \operatorname{div} \mathbf{G}-$

$-\mathbf{G} \operatorname{div} \mathbf{F}$, 
we obtain finally the formula for a force $\mathbf{F}$ acting on a volume element $d V$ of a spontaneously polarized liquid dielectric:

$\mathbf{F}=\nabla\left[-p+\left.\frac{E^{2}}{8 \pi} \rho \frac{\partial \varepsilon}{\partial \rho}\right|_{T}\right]-\frac{E^{2}}{8 \pi} \nabla \varepsilon+$

$+(a-1) \nabla\left(\mathbf{P}_{s} \mathbf{E}\right)+\left(\mathbf{P}_{s} \nabla\right) \mathbf{E}+\frac{1}{2} \operatorname{rot}\left(\mathbf{P}_{s} \times \mathbf{E}\right)$.

Three last terms arose due to the account for the spontaneous polarization.

Let us use the Clausius-Mossotti formula [5] $\frac{\varepsilon-1}{\varepsilon+2}=$ $=\frac{4 \pi}{3} n \beta$, which holds for nonpolar fluids and gases (here, $\beta$ is the polarizability of a molecule). For the gases and some fluids including He II [30], $\varepsilon$ is close to 1 . Then $\varepsilon-1 \approx 4 \pi n \beta$ and $\rho \partial \varepsilon / \partial \rho \approx \varepsilon-1$. Instead of (58), we obtain

$\mathbf{F}=-\nabla p+\frac{\varepsilon-1}{8 \pi} \nabla E^{2}+\left(\mathbf{P}_{s} \nabla\right) \mathbf{E}+$
$+(a-1) \nabla\left(\mathbf{P}_{s} \mathbf{E}\right)+\frac{1}{2} \operatorname{rot}\left(\mathbf{P}_{s} \times \mathbf{E}\right)$.

This formula differs from (41) by two additional last terms. In this case, the term $(a-1) \nabla\left(\mathbf{P}_{s} \mathbf{E}\right)$ disappears at $a=1$. Interestingly, the solution $\xi \approx 7.5(7 / 3) d_{0} \bar{r}_{0}\left(n(\mathbf{r}) / n_{0}\right)[19]$ corresponds namely to $a=1$. Such value of $a$ is a scaling property [19]. Therefore, it should hold for any nonpolar fluid.

\section{Conclusion}

We have proposed a method of description of a nonpolar isotropic spontaneously polarized dielectric that involves the induced polarization $\mathbf{P}_{i}(\mathbf{r})$ accompanying the bare spontaneous polarization $\mathbf{P}_{s}(\mathbf{r})$. In Appendix, we will give (as examples) several solutions for the electric field in a spontaneously polarized isotropic dielectric under different boundary conditions.

The author is grateful to A.S. Rybalko for the detailed discussion of the experiments [9]. I also thank A. Morozovska for the discussion of the problem. The present work was partially supported by the National Academy of Sciences of Ukraine (project No. 0116U003191).

\section{APPENDIX.}

\section{Examples of Solutions}

The below-presented examples can be useful for the comprehension of properties of the field $\mathbf{E}$ inside of a resonator in experiments similar to [8-12]. In those experiments, the boundary conditions (BCs) are complicated. We will consider more simple $\mathrm{BCs}$ and will see how the shape of a resonator and the presence of an external metallic shell affect the field $\mathbf{E}$ (in experiments [9], the resonator was placed in a metallic shell for the protection against external electric signals).

\section{Spontaneously polarized plate in vacuum}

Consider a dielectric plate, which occupies the space region $x, y \in[-L / 2, L / 2], z \in[-H / 2, H / 2]$. Let the plate have the uniform total polarization $\mathbf{P}=P \mathbf{i}_{z}$ (we suppose that $\mathbf{P}$ is known). The plate is surrounded by vacuum.

The knowledge of the solution for the field $\mathbf{E}$ in this system is essential for the experiments like [8-12] and for the ferroelectrics. In many works (including well-known ones [7, 26]), the field in such system was described by the formulae

$\mathbf{E}^{(\mathrm{int})}=-4 \pi \mathbf{P}, \quad \mathbf{E}^{(\mathrm{ext})}=0$,

where $\mathbf{E}^{(\mathrm{int})}$ and $\mathbf{E}^{(\mathrm{ext})}$ are the fields inside and outside of the plate, respectively. However, the solution of this problem is different.

The problem can be solved accurately, if we start from Eq. (18), where $\bar{\rho}(\mathbf{r})=0$ in the space outside of a dielectric. Equation (18) is true in the whole space. Therefore, BCs (24) are satisfied automatically. The solution of Eq. (18) is given by formula (30), where $V$ is the volume of a dielectric, and we should set $\varepsilon=1, \mathbf{P}_{s}=\mathbf{P}$. In this case, we obtain

$$
\begin{aligned}
& \varphi(\mathbf{r})=P \int_{-L / 2}^{L / 2} d \dot{x} \int_{-L / 2}^{L / 2} d \dot{y} \times \\
& \times\left(\frac{1}{\sqrt{(H / 2-z)^{2}+(x-\hat{x})^{2}+(y-\hat{y})^{2}}}-\right. \\
& \left.-\frac{1}{\sqrt{(H / 2+z)^{2}+(x-\hat{x})^{2}+(y-\hat{y})^{2}}}\right) .
\end{aligned}
$$

This solution has the properties $\varphi(x, y,-z)=-\varphi(x, y, z)$, $E_{x}(x, y,-z)=-E_{x}(x, y, z), E_{y}(x, y,-z)=-E_{y}(x, y, z)$, and $E_{z}(x, y,-z)==E_{z}(x, y, z)$. We have found potential (61) and the field $\mathbf{E}(\mathbf{r})$ numerically, see Figs. 1 and 2 (the field near the plate edge is found approximately; the field $E_{y}(\mathbf{r})$ is very weak at $y=0$ and is not shown in the figures). It is clear from the symmetry of the problem that $E_{x}(\mathbf{r})$ coincides with $E_{y}\left(\mathbf{r}^{\prime}\right)$, where $\mathbf{r}^{\prime}$ is obtained by the rotation of $\mathbf{r}$ around the axis $z$ by $\pm 90^{\circ}$. As is seen from the plots, the field $\mathbf{E}(\mathbf{r})$ is nonuniform and has a complicated structure. Inside the dielectric, $\mathbf{E} \approx-2 \pi \mathbf{P}=-2 \pi P \mathbf{i}_{z}$. More accurately, $E_{x}, E_{y} \neq 0$; therefore, the dependence $\mathbf{D}(\mathbf{E})$ has a tensor form $D_{j}=\sum_{l} \tilde{\varepsilon}_{j l} E_{l}$. Outside the dielectric, the field $\mathbf{E}(\mathbf{r})$ is rather strong near the dielectric and decreases, as the distance increases. The direct checking indicates that BCs (24) hold on all surfaces of the plate.

To verify the solution, we will find it by another method. We start from Eq. (18) in the form

$\triangle \varphi=-4 \pi \bar{\rho}$.

ISSN 2071-0194. Ukr. J. Phys. 2019. Vol. 64, No. 6 
The equation $\bar{\rho}(\mathbf{r})=-\operatorname{div} \mathbf{P}(\mathbf{r})$ implies that the polarization $\mathbf{P}=P \mathbf{i}_{z}(P=$ const $)$ is equivalent to the charges on the lower and upper faces of a dielectric with the surface densities $\sigma(z=$ $=-H / 2)=-P$ and $\sigma(z=H / 2)=P$, respectively. We assume that there are $N_{q}$ point charges $q$ on the lower face and $N_{q}$ charges equal to $-q$ on the upper face. In this case, $N_{q} q / L^{2}=$ $=-P, N_{q} \rightarrow \infty$. Then

$\bar{\rho}=\sum_{j=1}^{N_{q}} q \delta\left(x-x_{j}\right) \delta\left(y-y_{j}\right)\left[\delta\left(z+\frac{H}{2}\right)-\delta\left(z-\frac{H}{2}\right)\right]$,

where $x_{j}, y_{j} \in[-L / 2, L / 2]$. We consider the space to be a closed cube with the volume $V_{s}=\Lambda^{3}(\Lambda \gg L)$ and expand $\bar{\rho}$ and $\varphi$ in Fourier series:

$\bar{\rho}(\mathbf{r})=\frac{1}{V_{s}} \sum_{\mathbf{k}} \rho_{\mathbf{k}} e^{i \mathbf{k r}}, \quad \varphi(\mathbf{r})=\frac{1}{V_{s}} \sum_{\mathbf{k}} \varphi_{\mathbf{k}} e^{i \mathbf{k r}}$.

Relation (63) yields $\rho_{\mathbf{k}=0}=0$ and

$\rho_{\mathbf{k} \neq 0}=\int_{V_{s}} \bar{\rho}(\mathbf{r}) e^{-i \mathbf{k r}} d \mathbf{r}=q 2 i \sin \left(k_{z} H / 2\right) \sum_{j=1}^{N_{q}} e^{-i k_{x} x_{j}-i k_{y} y_{j}}$.

Passing to the integration and taking into account that $\frac{q}{\triangle x \triangle y}=-P$, we get

$\rho_{\mathbf{k} \neq 0}=-P 2 i \sin \left(\frac{k_{z} H}{2}\right) \int_{-\frac{L}{2}}^{\frac{L}{2}} d x \int_{-\frac{L}{2}}^{\frac{L}{2}} d y e^{-i k_{x} x_{j}-i k_{y} y_{j}}=$

$=-\frac{P 8 i}{k_{x} k_{y}} \sin \left(k_{z} H / 2\right) \sin \left(k_{x} L / 2\right) \sin \left(k_{y} L / 2\right)$.

At $k_{x}=0$, we set $\frac{\sin \left(k_{x} L / 2\right)}{k_{x}}=L / 2$ (analogously for $\left.k_{y}=0\right)$. Relation (62) yields $\varphi_{\mathbf{k} \neq 0}=4 \pi \rho_{\mathbf{k}} / k^{2}$. Thus, we have a solution for the potential:

$\varphi(\mathbf{r})=\varphi_{0}-\frac{32 \pi P}{\Lambda^{3}} \sum_{k_{x}} \frac{\sin \left(k_{x} L / 2\right)}{k_{x}} e^{i k_{x} x} \times$

$\times \sum_{k_{y}} \frac{\sin \left(k_{y} L / 2\right)}{k_{y}} e^{i k_{y} y} \sum_{k_{z}} \frac{i \sin \left(k_{z} H / 2\right)}{k_{x}^{2}+k_{y}^{2}+k_{z}^{2}} e^{i k_{z} z}$,

where $k_{x}=2 \pi j_{x} / \Lambda, k_{y}=2 \pi j_{y} / \Lambda, k_{z}=2 \pi j_{z} / \Lambda, j_{x}, j_{y}, j_{z}=$ $=0, \pm 1, \pm 2, \ldots$, and $k_{x}^{2}+k_{y}^{2}+k_{z}^{2} \neq 0$. At infinity $(z= \pm \Lambda / 2)$, we set $\varphi=0$. Since $\varphi(z= \pm \Lambda / 2)=\varphi_{0}$, we obtain $\varphi_{0}=0$. At $k_{\rho} \Lambda \gg 1$, the sum over $k_{z}$ in (67) can be found analytically:

$\left.I_{z}\right|_{k_{\rho} \neq 0}=\sum_{k_{z}} \frac{i \sin \left(k_{z} H / 2\right)}{k_{x}^{2}+k_{y}^{2}+k_{z}^{2}} e^{i k_{z} z}=$

$=\frac{1}{2} \sum_{k_{z}} \frac{\cos \left[k_{z}(H / 2+z)\right]-\cos \left[k_{z}(H / 2-z)\right]}{k_{\rho}^{2}+k_{z}^{2}}=$

$=\frac{\Lambda}{4 k_{\rho}}\left(e^{-k_{\rho}|z+H / 2|}-e^{-k_{\rho}|z-H / 2|}\right)$,

where $k_{\rho}=\sqrt{k_{x}^{2}+k_{y}^{2}}$. Therefore, one needs to present $\varphi(\mathbf{r})$ (67) in the form $\varphi\left(k_{\rho}=0, k_{z} \neq 0\right)+\varphi\left(k_{\rho} \neq 0\right)$, where $\varphi\left(k_{\rho} \neq\right.$

ISSN 2071-0194. Ukr. J. Phys. 2019. Vol. 64, No. 6

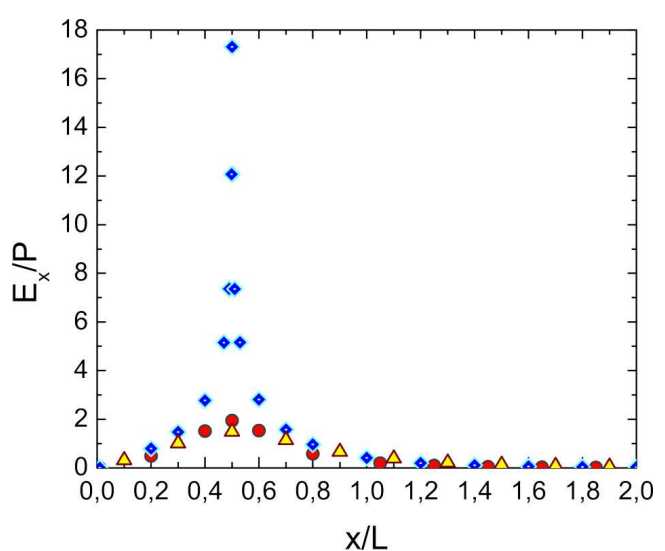

Fig. 1. [Color online] $E_{x}(x)$ for a dielectric plate with $L=$ $=2 H$ at $y=0$ and $z=H / 4$ (circles) (1), $z=H / 2$ (rhombs) (2), $z=H$ (triangles) (3). It is clear from the symmetry that the same curves correspond to $E_{y}(y)$ for $x=0$ and $z=$ $=H / 4 ; H / 2 ; H$. The lateral surface of the plate corresponds to $x / L=0.5$

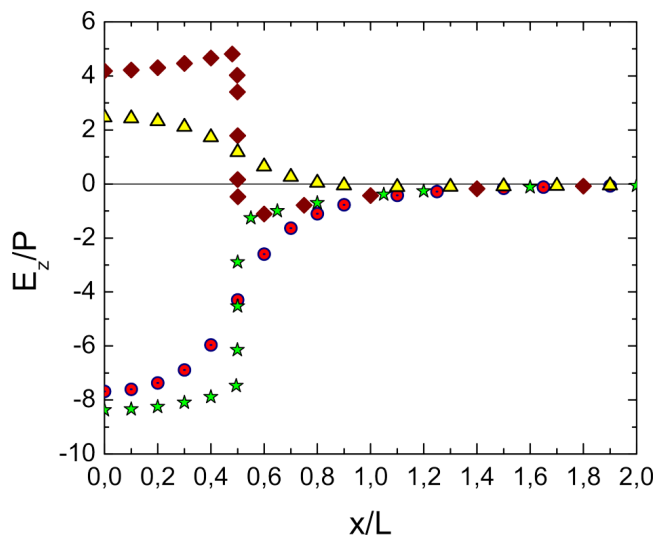

Fig. 2. [Color online] $E_{z}(x)$ for a dielectric plate with $L=$ $=2 H$ at $y=0$ and $z=H / 4$ (circles) (1), $z=0.499 H$ (stars) (2), $z=0.501 H$ (rhombs) (3), $z=H$ (triangles) (4). The symmetry implies that the function $E_{z}(y)$ at $x=0$ and $z=$ $=H / 4 ; 0.499 H ; 0.501 H ; H$ is described by the same curves. The upper surface of the plate corresponds to $z=0.5 H$. The stars and rhombs show the field at the very boundary (under and above the surface, respectively). The jump of $E_{z}$ on the upper surface is equal to $4 \pi P$ in agreement with BCs (24)

$\neq 0$ ) should be calculated with the help of (68). The numerical analysis shows that solutions (61) and (67) coincide with a good accuracy at $\Lambda \gtrsim 100 L$.

For an infinite plate, we set $L=\Lambda \rightarrow \infty$ in (67). Then only the terms with $k_{\rho}=0$ are nonzero in (67). Let $k_{\rho}=10^{2} / \Lambda$. Then formula (68) is the exact one, and $k_{\rho} \rightarrow 0$. We obtain

$\varphi(\mathbf{r})=-\left.\frac{8 \pi P}{\Lambda} I_{z}\right|_{k_{\rho} \rightarrow 0}=2 \pi P\left(\left|z+\frac{H}{2}\right|-\mid z-\frac{H}{2}\right)$, 
which leads to (60). We obtain the same result, by directly calculating sum (67). This agrees with the solution in [23]. Solution (67) transits smoothly into (60), as $L / H$ increases: for $L / H \gtrsim 100$, the solution is close to that in (60); for $L / H=\infty$, it coincides with (60). Thus, solution (60) is valid only in the case of infinite plate.

We note that the solution for a uniformly polarized cylinder of finite length was found in [24], and the solutions for uniformly polarized nanoparticles of different shapes were presented in [25].

In the examples given below, we consider that only $\mathbf{P}_{s}$ is known and take $\mathbf{P}_{i}$ into account separately.

\section{Spontaneously polarized dielectric ball in vacuum}

Let the ball of radius $R$ with the dielectric permittivity $\varepsilon$ be uniformly spontaneously polarized: $\mathbf{P}_{s}=P_{s} \mathbf{i}_{z}$. The spontaneous dipoles create the electric field, which produces the induced polarization

$\mathbf{P}_{i}=\frac{(\varepsilon-1) \mathbf{E}^{(\mathrm{int})}}{4 \pi}$.

Consider the system as a set of spontaneous and induced dipoles in vacuum. The field $\mathbf{E}$ is determined by Eq. (18). The solution for a domain inside the ball is [5]

$\mathbf{E}^{(\text {int })}=-\frac{4 \pi \mathbf{P}}{3}=-\frac{4 \pi\left(\mathbf{P}_{i}+\mathbf{P}_{s}\right)}{3}$

[see also Eq. (82) below, which is the solution of Eq. (23)]. Relations (69) and (70) yield

$\mathbf{P}_{i}=-\frac{\varepsilon-1}{\varepsilon+2} \mathbf{P}_{s}, \quad \mathbf{E}^{(\text {int })}(\mathbf{r})=-\frac{4 \pi}{\varepsilon+2} \mathbf{P}_{s}$,

$\varphi^{(\mathrm{int})}(\mathbf{r})=\frac{4 \pi}{\varepsilon+2} \mathbf{P}_{s} \mathbf{r}$.

Setting $4 \pi \mathbf{P}_{s}=\zeta \mathbf{E}^{(\text {int })}$, we have $\zeta=-\varepsilon-2, \tilde{\varepsilon}=\varepsilon+\zeta=-2$.

The field $\varphi^{(\text {ext })}(\mathbf{r})$ outside the ball is created by spontaneous and induced dipoles located inside the ball. This field can be determined in the following way. The uniformly polarized ball centered at the point $\mathbf{r}=0$ can be considered as two uniformly charged balls: the ball with charge $-Q<0$ centered at the point $\mathbf{r}_{-}=\left(x=y=0, z=-z_{0} / 2\right)$ and the ball with charge $Q$ centered at the point $\mathbf{r}_{+}=\left(x=y=0, z=z_{0} / 2\right)$, where $z_{0}$ is an infinitesimal value, and $Q z_{0}=P V=4 \pi P R^{3} / 3$. The ball with charge $-Q$ creates the potential $\varphi(\mathbf{r})=\frac{-Q}{\left|\mathbf{r}-\mathbf{r}_{-}\right|}$ around itself, and the ball with charge $Q$ generates the potential $\varphi(\mathbf{r})=\frac{Q}{\left|\mathbf{r}-\mathbf{r}_{+}\right|}$outside the ball. The total potential outside the polarized ball at $z_{0} \rightarrow 0$ is

$\varphi^{(\mathrm{ext})}(\mathbf{r})=\frac{-Q}{\left|\mathbf{r}-\mathbf{r}_{-}\right|}+\frac{Q}{\left|\mathbf{r}-\mathbf{r}_{+}\right|}=\frac{4 \pi R^{3}}{3} \frac{\mathbf{P r}}{r^{3}}=\frac{4 \pi R^{3}}{\varepsilon+2} \frac{\mathbf{P}_{s} \mathbf{r}}{r^{3}}$,

where $\mathbf{P}=\mathbf{P}_{s}+\mathbf{P}_{i}=\frac{3 \mathbf{P}_{s}}{\varepsilon+2}$. BCs (24) are satisfied.

We can solve the problem in a different way. If we set $\varphi^{(\text {int })}(\mathbf{r})=A \mathbf{P}_{s} \mathbf{r}, \varphi^{(\text {ext })}(\mathbf{r})=B \mathbf{P}_{s} \mathbf{r} / r^{3}$ and find the constants $A$ and $B$ from BCs (24), we obtain the same result.

\section{Spontaneously polarized} dielectric placed in a spherical conductor

Consider a grounded metallic sphere of radius $R_{m}$. Let it contain an isotropic dielectric with spontaneous dipoles $\mathbf{d}_{s}=$ $=\left|q_{0}\right| \mathbf{r}_{0}$ uniformly distributed over the volume and corresponding to the average spontaneous polarization $\mathbf{P}_{s}=n_{s} \mathbf{d}_{s}=P_{s} \mathbf{i}_{z}$ ( $n_{s}=$ const). The $\mathrm{BC}$ in the spherical coordinates $\rho, \theta, \phi$ reads $[3,4]$

$\varphi\left(\rho=R_{m}\right)=0$.

If the polarization $\mathbf{P}_{s}$ is perfectly uniform, then, for any shape of a resonator, the problem has the known solution

$\varphi(\mathbf{r})=0, \quad \mathbf{E}(\mathbf{r})=0$,

since the equation $\varepsilon \triangle \varphi=4 \pi \operatorname{div} \mathbf{P}_{s}$ with $\mathbf{P}_{s}=$ const and BCs (74) has the unique solution $\varphi(\mathbf{r})=0$. However, there is a difficulty: On the boundary, $\mathbf{P}_{s}$ decreases to zero by jump. Therefore, $\operatorname{div} \mathbf{P}_{s}=\infty$. The solution $\varphi(\mathbf{r})=0$ neglects this property. Below, we will find the solution within the method, which allows us to avoid this difficulty.

It was assumed in some works that the relation $\mathbf{D}(\mathbf{r})=0$ holds for a dielectric surrounded by a metallic shell. It was not substantiated or was substantiated by that the equality $\mathbf{D}(\mathbf{r})=0$ holds in a metal. In our opinion, this reasoning is incorrect. It is well known that a metal should be considered as a dielectric with $\varepsilon=\infty$ (see [2] Chapt. IV, §6; [4] Chapt. II, $\S 7$ and Problem 1 after $\S 7)$. In this case, $\mathbf{D}(\mathbf{r}) \neq 0$ inside a metal. In addition, the condition $\mathbf{D}(\mathbf{r})=0$ for dielectrics leads to the mathematical contradiction. Indeed, the equality $\mathbf{D}(\mathbf{r})=0$ yields $D_{n}=0$ on the surface. If $\varphi$ is given on a closed surface of the dielectric, then we can find a solution of the equation $-\operatorname{div}[\varepsilon(\mathbf{r}) \nabla \varphi(\mathbf{r})]=4 \pi \bar{\rho}(\mathbf{r})$ (with $\bar{\rho}(\mathbf{r})$ to be known) inside the dielectric uniquely $[2,3]$. This was proved for $\varepsilon=\mathrm{const}$ ([3], Chapt. 1, §9) and for $\varepsilon=\varepsilon(\mathbf{r})$ ([2], Chapt. III, §11). If we supplement Eq. (74) by the BC $D_{n}=0$, then the problem becomes overspecified and has no solutions for $\varphi(\mathbf{r})$.

Thus, we need to solve Eq. (26) with BC (74). The solution for the potential is given by formula (30). In order to satisfy (74), we need to consider "images" that are the reflections of dipoles in the metal. This is the most complicated part in problems of this kind. In our case, the simplest way to consider the images is, apparently, the following. A real uniformly polarized ball can be represented as two balls with radius $R$ shifted relative each other by the size $r_{0}$ of the dipole. The first ball is uniformly charged negatively (so that its total charge is $Q_{-}=N_{s} q_{0}=Q_{0}$, where $N_{s}$ is the total number of spontaneous dipoles in helium). Let its center have the coordinates $x=y=0, z=-r_{0} / 2$. The second ball is charged positively and has the total charge $Q_{+}=-Q_{-}$. The coordinates of its center are $x=y=0, z=r_{0} / 2$. The center of the segment joining both balls is the coordinate origin: $x=y=z=0$. We consider that these two balls are placed in a metallic sphere so that the $(-)$ and $(+)$ balls touch the internal surface of the sphere. In this case, $R_{m}=R+r_{0} / 2$. The sphere has contact with the balls at two opposite points, and the remaining points 
of the sphere are separated from two balls by a thin layer (with a thickness of $\lesssim r_{0} / 2$ ) of vacuum. The potential created by a uniformly charged dielectric ball of radius $R$ at a point located at the distance $R_{0}$ from the ball center is

$$
\begin{aligned}
& \varphi\left(\mathbf{R}_{0}\right)=\int_{V} \frac{(\varepsilon)^{-1} g d x d y d z}{\sqrt{\left(x-X_{0}\right)^{2}+\left(y-Y_{0}\right)^{2}+\left(z-Z_{0}\right)^{2}}}= \\
& =\int_{0}^{R} r^{2} d r \int_{0}^{\pi} \sin \theta d \theta \int_{0}^{2 \pi} \frac{(\varepsilon)^{-1} g d \phi}{\sqrt{r^{2}+R_{0}^{2}-2 r R_{0} \cos \theta}}= \\
& =\frac{2 \pi g}{\varepsilon R_{0}} \int_{0}^{R} r d r\left(r+R_{0}-\left|r-R_{0}\right|\right) .
\end{aligned}
$$

This formula yields

$$
\varphi\left(\mathbf{R}_{0}\right)=\left[\begin{array}{ll}
\frac{2 \pi g}{\varepsilon}\left(R^{2}-\frac{R_{0}^{2}}{3}\right), & R_{0} \leq R, \\
\frac{Q}{\varepsilon R_{0}}, & R_{0} \geq R .
\end{array}\right.
$$

Here, $g=Q / V=3 Q /\left(4 \pi R^{3}\right)$ is the charge density, and $\varepsilon$ is the dielectric permittivity of the ball. The dielectric weakens the field by $\varepsilon$ times in accordance with Eq. (26). Solution (77) is well known [5].

All points at a distance of $r \leq R_{m}-r_{0}$ from the coordinate origin belong to both balls: (-)-ball and (+)-ball. Let us consider this domain. According to (77), these two balls create at the point $\mathbf{r}$ the potential

$\varphi(\mathbf{r})=\frac{2 \pi g_{-}}{\varepsilon}\left(R^{2}-\frac{r_{-}^{2}}{3}\right)+\frac{2 \pi g_{+}}{\varepsilon}\left(R^{2}-\frac{r_{+}^{2}}{3}\right)$.

Here, $g_{-}=Q_{0} / V, g_{+}=-g_{-}, V=(4 \pi / 3) R^{3}$, and $r_{-}, r_{+}$are the distances from the point of observation $\mathbf{r}$ to the centers of the $(-)$ and $(+)$ balls, respectively:

$r_{-}^{2}=r^{2}+\left(r_{0} / 2\right)^{2}-r r_{0} \cos (\pi-\theta)$,

$r_{+}^{2}=r^{2}+\left(r_{0} / 2\right)^{2}-r r_{0} \cos \theta$.

In this case, the vector $\mathbf{r}$ is directed from the coordinate origin to the point of observation, and $\theta==\left(\widehat{\mathbf{i}_{z}, \mathbf{r}}\right)=\left(\widehat{\mathbf{r}_{0}, \mathbf{r}}\right)$. Formulae (78)-(80) yield the exact solution

$\varphi(\mathbf{r})=-\frac{Q_{0} r r_{0} \cos \theta}{\varepsilon R^{3}}=-\frac{Q_{0} \mathbf{r r} \mathbf{r}_{0}}{\varepsilon R^{3}}=\frac{4 \pi \mathbf{P}_{s} \mathbf{r}}{3 \varepsilon}$,

which is also well known [5] (in [5], formula (81) was deduced without $\varepsilon$, since the response of the medium to an external field was calculated). It is essential that relation (81) follows directly from formula (30). It is easy to see, making use of relations (30), (76), and (77). Relation (81) implies that, in the domain $r \leq R_{m}-r_{0}$, the electric field strength is constant:

$\mathbf{E}(\mathbf{r})=-\nabla_{\mathbf{r}} \varphi(\mathbf{r})=\frac{Q_{0} \mathbf{r}_{0}}{\varepsilon R^{3}}=-\frac{4 \pi \mathbf{P}_{s}}{3 \varepsilon}$.

According to (77), the $(-)$ and $(+)$ balls located inside the metallic sphere create the potential

$\varphi(\mathbf{r})=\frac{Q_{-}}{\varepsilon r_{-}}+\frac{Q_{+}}{\varepsilon r_{+}}$

ISSN 2071-0194. Ukr. J. Phys. 2019. Vol. 64, No. 6 on the internal surface of the sphere. It coincides with the potential, which is obtained, if the (-)-ball and the $(+)$-ball are replaced by the point charges $Q_{-}$and $Q_{+}$located at the centers of the $(-)$ and $(+)$ balls, respectively. In this case, $\mathrm{BC}(74)$ is easily satisfied: potential (83) can be exactly compensated on the whole surface of the cavity, if we introduce two additional charges that are the images of the point charges $Q_{-}$and $Q_{+}$.

Let the point charge $Q_{-}$be placed inside a conducting sphere of radius $R_{m}$ at a distance of $r_{0} / 2$ from its center. It is known (see [4], Chapt. I) that the image of such charge is located at a distance of $l_{-}=2 R_{m}^{2} / r_{0}$ from the sphere center, and the charge of this image is $q_{-}=-Q_{-} 2 R_{m} / r_{0}$. Moreover, the sphere center, charge, and image are located on the same line, and the charge is placed between the sphere center and the image. The potential created by the charge $Q_{-}$and its image is equal to zero on the whole surface of the sphere, which can be directly verified.

The $(-)$ and $(+)$ balls induce polarization charges on the internal surface of the metallic sphere. The field created by these charges inside the sphere coincides with the field of images. With regard for this, the total potential at a point $\mathbf{r}$ inside the sphere is the sum of potentials created at this point by the (-)-ball, (+)-ball, and images of the point charges $Q_{-}$and $Q_{+}$. The solution for $r \leq R_{m}-r_{0}$ is as follows:

$\varphi(\mathbf{r})=-\frac{Q_{0} r r_{0} \cos \theta}{\varepsilon R^{3}}+\frac{q_{-}}{\varepsilon r_{q_{-}}}+\frac{q_{+}}{\varepsilon r_{q_{+}}}=$

$=-\frac{Q_{0} r r_{0} \cos \theta}{\varepsilon R^{3}}-\frac{2 R_{m} Q_{0}}{\varepsilon r_{0} r_{q_{-}}}+\frac{2 R_{m} Q_{0}}{\varepsilon r_{0} r_{q_{+}}}$.

Here, $r_{q_{-}}$and $r_{q_{+}}$are the distances from the point of observation $\mathbf{r}$ to the images of the charges $Q_{-}$and $Q_{+}$, respectively:

$r_{q_{-}}^{2}=r^{2}+\left(l_{-}\right)^{2}-2 r l_{-} \cos (\pi-\theta)$,

$r_{q_{+}}^{2}=r^{2}+\left(l_{+}\right)^{2}-2 r l_{+} \cos \theta$,

where $l_{-}=2 R_{m}^{2} / r_{0}=L$ and $l_{+}=2 R_{m}^{2} / r_{0}=L$ are the distances from the image of the charge $Q_{-}$and the image of the charge $Q_{+}$to the center of the spherical cavity. Since the charges in (83) are decreased by $\varepsilon$ times, the charges of the images in (84) are also decreased by $\varepsilon$ times.

At $r_{0} / R \ll 1, r \leq R_{m}$, we have $r / L \ll 1$. Let us use $r / L$ as a small parameter. Then relations (85) and (86) yield

$\frac{1}{r_{q_{-}}}=\frac{1}{L}\left[1-\frac{r \cos \theta}{L}+\frac{r^{2}}{L^{2}}\left(\frac{3}{2} \cos ^{2} \theta-\frac{1}{2}\right)+\right.$

$\left.+\frac{r^{3}}{L^{3}}\left(\frac{3}{2} \cos \theta-\frac{5}{2} \cos ^{3} \theta\right)+O\left(\frac{r^{4}}{L^{4}}\right)\right]$,

$\frac{1}{r_{q_{+}}}=\frac{1}{L}\left[1+\frac{r \cos \theta}{L}+\frac{r^{2}}{L^{2}}\left(\frac{3}{2} \cos ^{2} \theta-\frac{1}{2}\right)+\right.$

$\left.+\frac{r^{3}}{L^{3}}\left(\frac{5}{2} \cos ^{3} \theta-\frac{3}{2} \cos \theta\right)+O\left(\frac{r^{4}}{L^{4}}\right)\right]$.

Substituting expansions (87) and (88) in (84) and taking the relation $L=2 R_{m}^{2} / r_{0}$ into account, we get

$\varphi(\mathbf{r})=\frac{Q_{0} r r_{0} \cos \theta}{\varepsilon}\left(\frac{1}{R_{m}^{3}}-\frac{1}{R^{3}}\right)+$ 
$+\frac{Q_{0} r^{3} r_{0}^{3}}{8 R_{m}^{7} \varepsilon}\left(5 \cos ^{3} \theta-3 \cos \theta\right)+O\left(\frac{r^{5}}{L^{5}}\right)$.

In view of the formulae $r r_{0} \cos \theta=\mathbf{r r}_{0}, R_{m}=R+r_{0} / 2$ and the smallness of $r_{0} / R$, relation (89) yields finally:

$\varphi(\mathbf{r})=-\frac{Q_{0} \mathbf{r r}_{0}}{\varepsilon R^{3}} \frac{3 r_{0}}{2 R}+O\left(\frac{r_{0}^{3}}{R^{3}}\right)$,

$\mathbf{E}(\mathbf{r})=-\nabla_{\mathbf{r}} \varphi(\mathbf{r}) \approx \frac{Q_{0} \mathbf{r}_{0}}{\varepsilon R^{3}} \frac{3 r_{0}}{2 R}=-\frac{2 \pi \mathbf{P}_{s}}{\varepsilon} \frac{r_{0}}{R}$.

Two last formulae imply that the spherical conductor decreases potential (81) and the field strength (82) approximately by $R / r_{0}$ times. For example, the known mechanisms of polarization of He II give the value of $r_{0}$ comparable with (or much less of) the interatomic distance. Taking the realistic values $R \sim 1 \mathrm{~cm}$ and $r_{0} \sim 10 \AA$, we get $R / r_{0} \sim 10^{7}$. In other words, the images almost completely suppress the electric field $\mathbf{E}$ inside a spontaneously polarized dielectric ball.

We remark that, at $r_{0}=0$, the dielectric possesses a perfectly uniform polarization. In this case, relations (90) and (91) yield solution (75). However, for real bodies, $r_{0}$ is small, but nonzero (since $r_{0}$ is the size of an elementary dipole, e.g., a molecule). Therefore, near the boundary, $\mathbf{P}$ is nonuniform in a layer of finite thickness $\sim r_{0}$. The result given by (90) and (91) is apparently new.

Formula (91) and the relation $4 \pi \mathbf{P}_{s}=\zeta \mathbf{E}$ yield $\zeta=$ $=-2 \varepsilon R / r_{0}$. The dielectric coefficient $\zeta$ turns out to be very large in modulus, which leads to the smallness of $\mathbf{E}$. In this case, $\mathbf{D}=(\varepsilon+\zeta) \mathbf{E}=\varepsilon \mathbf{E}\left(1-2 R / r_{0}\right)$.

We now determine the field inside a spherical conductor in a thin layer of width $r_{0}$ near the sphere surface. This layer is divided into two regions: the region lying outside the (-)-ball and inside the $(+)$-ball (or vice versa) and the region lying outside the $(-)$ and $(+)$ balls.

For the region outside the $(-)$-ball and inside the $(+)$-ball, the above formulae give

$$
\begin{aligned}
& \varphi(\mathbf{r})=\frac{2 \pi g_{-}}{\varepsilon}\left(R^{2}-\frac{r_{-}^{2}}{3}\right)+\frac{Q_{+}}{\varepsilon r_{+}}+\frac{q_{-}}{\varepsilon r_{q_{-}}}+ \\
& +\frac{q_{+}}{\varepsilon r_{q_{+}}} \approx \frac{3 Q_{0}}{2 \varepsilon R}+\frac{Q_{0} \mathbf{r r}_{0}}{2 \varepsilon R^{3}}\left(1-\frac{3 r_{0}}{R}\right)- \\
& -\frac{Q_{0} \mathbf{r r}_{0}}{2 \varepsilon r^{3}}-\frac{Q_{0}\left(4 r^{2}+r_{0}^{2}\right)}{8 \varepsilon R^{3}}-\frac{Q_{0}}{\varepsilon r}- \\
& -\frac{Q_{0} r_{0}^{2}\left(3 \cos ^{2} \theta-1\right)}{8 \varepsilon r^{3}}+O\left(\frac{r_{0}^{3}}{R^{3}}\right), \\
& \mathbf{E}(\mathbf{r}) \approx-\frac{3 Q_{0}\left(\mathbf{r r}_{0}\right) \mathbf{i}_{\mathbf{r}}}{2 \varepsilon r^{4}}+\frac{Q_{0} \mathbf{i}_{\mathbf{r}}\left(r^{3}-R^{3}\right)}{\varepsilon R^{3} r^{2}}, \mathbf{i}_{\mathbf{r}}=\frac{\mathbf{r}}{r} .
\end{aligned}
$$

Since $r \approx R$ for this region, it is seen that the strength $\mathbf{E}$ is comparable by magnitude with strength (82) of the problem without a resonator.

For the region between the dielectric and the conductor (outside the $(-)$ and $(+)$ balls), we have

$$
\begin{aligned}
& \varphi(\mathbf{r})=\frac{Q_{-}}{\varepsilon r_{-}}+\frac{Q_{+}}{\varepsilon r_{+}}+\frac{q_{-}}{\varepsilon r_{q_{-}}}+\frac{q_{+}}{\varepsilon r_{q_{+}}} \approx \\
& \approx \frac{Q_{0} \mathbf{r r}_{0}}{\varepsilon R_{m}^{3}}-\frac{Q_{0} \mathbf{r r}_{0}}{\varepsilon r^{3}},
\end{aligned}
$$

$\mathbf{E}(\mathbf{r}) \approx-\frac{Q_{0} \mathbf{r}_{0}}{\varepsilon R_{m}^{3}}+\frac{Q_{0} \mathbf{r}_{0}}{\varepsilon r^{3}}-\frac{3 Q_{0}\left(\mathbf{r r}_{0}\right) \mathbf{i}_{\mathbf{r}}}{\varepsilon r^{4}}$

Here, the field strength is also comparable with (82). In this case, strength (93) is directed radially, (95) has the radial and $z$ components, and strength (82) is directed along the $z$-axis.

In the region $r \leq R_{m}$, the potential is continuous, whereas the field strength undergoes a jump on the surface of the dielectric and the internal surface of the conductor. It is seen from (94) that BC (74) is satisfied.

Thus, the electric field is strong (comparable with the field in the absence of a conductor) only in the narrow space of thickness $\leq r_{0}$ near the internal surface of the conductor. In the remaining volume inside the conductor (i.e., in almost whole volume of the dielectric), the field $\mathbf{E}$ is almost completely suppressed. This effect is caused by the presence of the conductor around the dielectric. It has a simple visual explanation. Without a conductor, the field $\mathbf{E}$ inside the dielectric is uniform (see (82)). The field created by the conductor in the region $r \leq R_{m}$ coincides with the field of images. But the images are remote from the dielectric by the distance $L=2 R_{m}^{2} / r_{0}$, which is much larger than the size $2 R$ of the dielectric. Therefore, the field created by the images inside the dielectric is almost uniform, but is directed against the intrinsic field (82) of the dielectric and compensates it. If the compensation would be absent, then the condition $\varphi=0$ would not be satisfied: the condition $\varphi=$ const requires $\mathbf{E}_{t}=0$ on the internal surface of the conductor. Since the field $\mathbf{E}$ is uniform inside the dielectric, the condition $\mathbf{E}_{t}=0$ on the surface requires that $\mathbf{E} \approx 0$ in the whole volume of the dielectric.

Note that a decrease in the electric energy of the dielectric because of the location of this dielectric in a spherical conductor is equal to the work (with opposite sign) that must be performed in order to transport two metallic hemispheres from infinity and to enclose a polarized dielectric by these hemispheres.

As a rule, a dielectric is in the gravity field. Therefore, such dielectric is slightly spontaneously polarized. If the walls of a vessel are vertical, and the bottom is horizontal, the polarization is uniform. In this case, the electric field is weak, but measurable [19]. However, if the shape of a vessel is a sphere, then the polarization should have the $z$ - and $\mathbf{r}$-components and, therefore, should be nonuniform. In other words, it is apparently impossible to get the uniform spontaneous polarization $\mathbf{P}_{s}$ in a spherical vessel.

We mentioned above that, in experiments [9], the resonator was placed in a metallic shell. The polarization of helium in those experiments is due to the second sound and, therefore, should be nonuniform. In this case, we expect that the shell weakens the field $\mathbf{E}$ only by several times or by one order of magnitude.

1. J.C. Maxwell. A Treatise on Electricity and Magnetism (Clarendon Press, 1892).

2. W.R. Smithe. Static and Dynamic Electricity (McGrawHill, 1950).

ISSN 2071-0194. Ukr. J. Phys. 2019. Vol. 64, No. 6 
3. J.D. Jackson. Classical Electrodynamics (Wiley, 1962) [ISBN: 0471431311, 978-0471431312].

4. L.D. Landau, E.M. Lifshitz. Electrodynamics of Continuous Media (Pergamon Press, 1984) [ISBN: 9780080302751].

5. I.E. Tamm. Fundamentals of the Theory of Electricity (Mir, 1979) (in Russian), Chapt. II [ISBN: 5-9221-0313$\mathrm{X}]$.

6. M. Born, K. Huang. Dynamical Theory of Crystal Lattices (Clarendon Press, 1988) [ISBN: 0198503695, 9780198503699].

7. B.A. Strukov, A.P. Levanyuk. Ferroelectric Phenomena in Crystals: Physical Foundations (Springer, 1998) [ISBN: 978-3-642-64340-8].

8. A.S. Rybalko, S.P. Rubets. Observation of mechanoelectric effect in He II. Low Temp. Phys. 31, 623 (2005).

9. A.S. Rybalko. Observation of the electric induction due to a second-sound wave in He II. Low Temp. Phys. 30, 994 (2004).

10. T.V. Chagovets. A study of the electric response of He II at the excitation of second sound waves. Low Temp. Phys. 42, 176 (2016).

11. H. Yayama, Y. Nishimura, H. Uchiyama, H. Kawai, J.-P. van Woensel, A.G. Hafez. Electric response induced by second sound in superfluid helium. Low Temp. Phys. 44, 1090 (2018).

12. T.V. Chagovets. Detection of electric response at excitation of first sound in He II. J. Low Temp. Phys. 187, 383 (2017).

13. A.K. Tagantsev. Pyroelectric, piezoelectric, flexoelectric, and thermal polarization effects in ionic crystals. Sov. Phys. Usp. 30, 588 (1987).

14. V.L. Ginzburg. Phase transitions in ferroelectrics: some historical remarks. Physics-Uspekhi 44, 1037 (2001).

15. E.A. Eliseev, A.N. Morozovska. General approach for the description of size effects in ferroelectric nanosystems, J. Mater. Sci. 44, 5149 (2009).

16. W. Byers Brown, D.M. Whisnant. Interatomic dispersion dipole. Mol. Phys. 25, 1385 (1973).

17. D.M. Whisnant, W. Byers Brown. Dispersion dipole between rare-gas atoms. Mol. Phys. 26, 1105 (1973)

18. V.M. Loktev, M.D. Tomchenko. On the mutual polarization of two $\mathrm{He}^{4}$ atoms. J. Phys. B: At. Mol. Opt. Phys. 44, 035006 (2011).

19. M.D. Tomchenko. Some mechanisms of "spontaneous" polarization of superfluid He-4. J. Low Temp. Phys. 158, 854 (2010).

20. L.D. Landau, E.M. Lifshitz. The Classical Theory of Fields (Pergamon Press, 1971), Chapt. V [ISBN: 9780080160191].
21. M.D. Tomchenko. Theory of the electric activity of He II induced by waves of first and second sound. Phys. Rev. B 83, 094512 (2011).

22. G.A. Korn, T.M. Korn. Mathematical Handbook for Scientists and Engineers: Definitions, Theorems, and Formulas for Reference and Review (McGraw-Hill, 1968).

23. R. Kretschmer, K. Binder. Surface effects on phase transitions in ferroelectrics and dipolar magnets. Phys. Rev. B 20, 1065 (1979).

24. A.N. Morozovska, E.A. Eliseev, M.D. Glinchuk. Size effects and depolarization field influence on the phase diagrams of cylindrical ferroelectric nanoparticles. Physica B 387, 358 (2007).

25. E.A. Eliseev, A.V. Semchenko, Y.M. Fomichov, M.D. Glinchuk, V.V. Sidsky, V.V. Kolos, Yu.M. Pleskachevsky, M.V. Silibin, N.V. Morozovsky, A.N. Morozovska. Surface and finite size effects impact on the phase diagrams, polar, and dielectric properties of $(\mathrm{Sr}, \mathrm{Bi}) \mathrm{Ta}_{2} \mathrm{O}_{9}$ ferroelectric nanoparticles. J. Appl. Phys. 119, 204104 (2016).

26. V.L. Ginzburg. Theory of ferroelectric phenomena, Uspekhi Fiz. Nauk 38, 490 (1949) (in Russian).

27. J.W. Gibbs. Elementary Principles in Statistical Mechanics (Scribner's sons, 1902), Chapters I, IV.

28. N.N. Bogoliubov. Lectures on Quantum Statistics, vol. 1: Quantum Statistics (Gordon and Breach, 1967) [ISBN: 0677200307, 9780677200309].

29. L.D. Landau, E.M. Lifshitz. Theory of Elasticity (Pergamon Press, 1970) [ISBN: 978-0080064659].

30. B.N. Esel'son, V.N. Grigor'ev, V.G. Ivantsov, E.Ya. Rudavskii, D.N. Sanikadze, I.A. Serbin. Properties of Liquid and Solid Helium (Izd. Standartov, 1978) (in Russian).

Received 19.11.18

М.Д. Томченко

ЕЛЕКТРИЧНЕ ПОЛЕ ТА ЕЛЕКТРИЧНІ СИЛИ У СПОНТАННО ПОЛЯРИЗОВАНОМУ НЕПОЛЯРНОМУ ІЗОТРОПНОМУ ДІЕЛЕКТРИКУ

$\mathrm{P}$ е $з$ ю м е

Виходячи з мікроскопічних рівнянь Максвелла, ми будуємо метод опису електричного поля в спонтанно поляризованому ізотропному неполярному діелектрику. Ми знаходимо розв'язок для електричного поля $\mathbf{E}(\mathbf{r})$ для кількох характерних прикладів. Крім того, ми узагальнюємо формулу Гельмгольця для електричної сили, яка діє на елемент об'єму діелектрика, враховуючи внесок спонтанної поляризації. 